\title{
ENHANCING THE TRANSFERABILITY OF HEALTH PROMOTION POLICIES TO BULGARIA: APPLICATION OF A MODEL TO INCREASE COMMUNICATION EFFECTIVENESS
}

\author{
M. Warner ${ }^{1}$, A. Kerekovska ${ }^{2}$ \\ ${ }^{1}$ University of Glamorgan, UK, ${ }^{2}$ Medical University “Prof. Dr. Paraskev Stoyanov", Varna \\ Reviewed by: Assoc. prof. S. Popova, PhD
}

SUMMARY

Having a healthy population is vital for economic development. This has been recognized over many decades by countries across the developed world, including Bulgaria. But there are major threats - tobacco consumption, obesity, heart disease and the like, which are the subject of attention by the international health promotion community. This community, and the policies they produce, is dominated by the cultures which make up Western Europe, North America and Australasia. A major question in the rest of the world is whether these 'global' policies will 'travel' well; and what might be done, in communication terms, to enhance their transferability. This paper reports on research carried out in Bulgaria. It began with an analysis of the contents of the major western-based international health promotion policy documents to identify expressions or ideas, contained in them that might be 'alien' to the culture of Bulgaria, as identified by Hofstede's Model of National Cultures. Through the use of a Delphi panel the international language was desensitised. A before and after quasi-experimental design was employed across three types of communities - urban, rural and tobacco-producing - to measure the degree to which communication was more effective using the desensitized material. These results, in most cases, showed a significant improvement. As Bulgaria joins the EU in January 2007 this study takes on broader importance. There will be many new policies to which adjustment is required at a national level; and true citizenship of Europe is dependent upon Bulgarians as a whole being able to identify with the intentions of European policies. Health promotion is only the beginning!

Keywords: health promotion policy; international cross-cultural transfer; Bulgaria

\section{INTRODUCTION}

Having a healthy population is vital for economic development. This has been recognized over many decades by countries across the developed world, including Bulgaria; and recent empiric work supports the idea (5). But there are major global threats - tobacco consumption, obesity, heart disease, environmental pollution and the like, which are the subject of attention by the international health promotion community. This community and the policies they produce are dominated by the cultures, which make up Western Europe, North America and Australasia. A major question in the rest of the world is whether these 'global' policies will 'travel' well; and what might be done, in communication terms, to enhance their transferability.

International health promotion policies and their consistent implementation on a national and sub-national level are important for addressing the threats. But the principles under-

Address for correspondence:

Morton Warner, Director, Welsh Institute for Health \& Social Care, University of Glamorgan, Pontypridd CF37 IDL, Wales, UK

E-mail: mmwarner@glam.ac.uk pinning much of the global health promotion can challenge national cultures and value systems, and affect their applicability to culturally distinctive contexts. Dominated by western values and approaches, they cannot simply be exported with any great hope of success to non-westernized settings which do not share the same values and have different cultural patterns. The policy language is not immediately and easily transferable, for instance, to Eastern European countries where the connotations may be politically sensitive at worst, or not culturally and socially aligned at best (4). It is, therefore, essential to improve the transfer of health promotion global policies to enhance their impact on a local level.

This study explores the possibility of enhancing the transmission of global health promotion policy messages into culturally diverse localities by considering it as a 'process of communication'. The main question is: "Whether by making the language of international health promotion messages more culturally specific, their communication at a local level can be improved and their impact within a country can be enhanced?"

Bulgaria has made remarkable progress over recent years in its attempts to integrate with the European Union. As an ac- 
cession country, it is in a process of alignment in all areas of society, including health and health promotion policy.

The development of strategies and programmes compatible with the global health promotion policy ideas is of extreme importance in the context of the country's 'full' accession into the EU.

Hofstede's Work on National Culture - The Essentials Using the Hofstede Model of National Cultures (3) reveals the substantial cultural specificity of health promotion fundamental principles and concepts. Given they have been articulated on the basis of a specific cultural pattern (a combination of 'small power distance' and 'weak uncertainty avoidance' as defined by Hofstede), the potential for cross-cultural transfer of policy ideas in this area is weakened. For example, the empowerment approach, participation, partnership formation, and intersectorality - essential elements of health promotion practice - correspond to the cultural pattern of Western Europe and North America, characterized by short decision hierarchies, flexible rules and high levels of negotiation. The Hofstede appraisal showed these fundamental principles of health promotion were not readily applicable to the specific cultural pattern of Bulgaria. Determined by 'large power distance' and 'strong uncertainty avoidance' characteristics, Bulgarian national culture is more hierarchical and involves unity of command and values and the concentration of authority and structures. This does not seem to favour comprehension of some of the essential western-developed components of international health promotion policy language and does not provide a suitable context for direct application of health promotion global principles and concepts. Therefore, through the contextual interpretation of global policy language and its specification to the cultural pattern of Bulgaria, this study aimed to significantly enhance the acceptability of in- ternational health promotion messages to the Bulgarian context.

\section{MATERIALS AND METHODS}

The research began with an analysis of the contents of the major western-based international health promotion policy documents (such as the Ottawa (7) Charter, Adelaide (8) and Jakarta Declarations (9)) to identify expressions or ideas, contained in them that might be 'alien' to the culture of Bulgaria, as identified by Hofstede's model. Statements containing the identified culturally-loaded words and phrases were extracted from the source policy documents and translated initially into literal Bulgarian. Then, proposals were made for an interpretation of the international health messages (western language and ideas) that would make them more culturally acceptable, and thus locally workable and applicable in the Bulgarian locations, whilst still maintaining their original intent. Through a Delphi panel agreement was achieved for a desensitised and contextualized version for Bulgaria context.

The transmission of international policy messages to localities was considered a 'process of communication,' and a Model of Communication Effectiveness $(2,6)$ (See Figure 1.) was applied as an operational framework. The assumption made was that the effectiveness of transmission of global policy messages to localities is very much dependant on local sensitivities to the international policy language (a measure of 'Social Distance' between recipients and senders of messages), and the level of importance attached to the messages by recipients (referred to as 'Message Magnitude'). The tangent of the angle created by the ratio of these two components represented a measure of effectiveness of

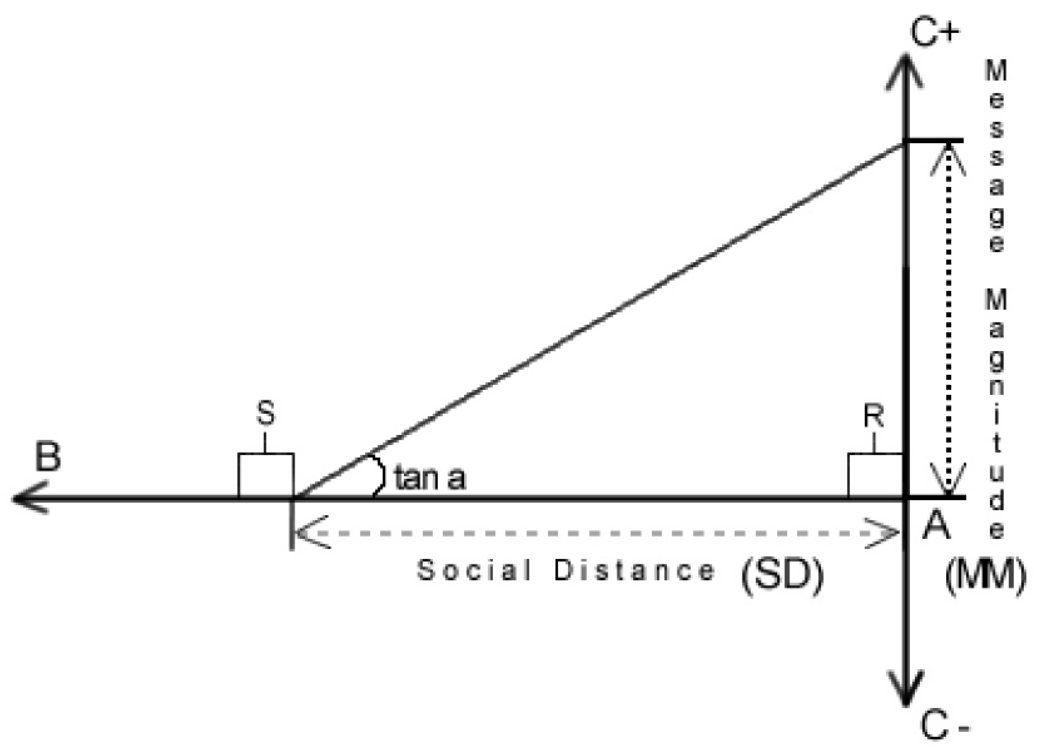

Source: A. Foot (1970)
AB - Continuum of Social

Distance

AC+ - Continuum of

Message Magnitude

positive

AC - - Continuum of

Message Magnitude

negative

$\mathbf{R}$ - Receiver of message

S - Sender of message;

tan $\boldsymbol{a}$ - Angle of

Communication

Effectiveness

SD - Social Distance

MM - Message Magnitude

Figure 1. The Model of Communication Effectiveness 
Enhancing the transferability of health promotion policies to Bulgaria: ...

communicating the policy messages - 'Communication Effectiveness'. The policy 'sender' in the case of this 'communication process' was represented by WHO with its global (western-based) international health promotion policy documents. The 'recipients' of the international policy messages in this instance were local implementers of health promotion policy - elected legislators and municipal councillors from Bulgarian localities.

Through the cultural specification and contextual adaptation of policy language it was aimed to reduce the 'Social Distance' between recipients and senders of health promotion messages (i.e. reduce the sensitivity to policy language) and thus enhance their 'Communication Effectiveness' (i.e. the acceptability) of international policy messages within Bulgaria.

To see if the 'Social Distance' is reduced significantly in respect of selected fundamental health promotion statements, and to measure the degree to which communication was more effective using the desensitized material,

a before and after ('Equivalent Materials Samples') approach was employed across three types of communities urban, rural and tobacco-producing and within three different health promotion subject areas - 'health promotion-general', 'environment' and 'tobacco control'. The study involved 158 respondents - local politicians and municipal technique during the time of the regular Municipal Council sessions in May and June 2003.

\section{RESULTS AND DISCUSSION}

The results showed that the adaptation language can bring significant decrease in local sensitivity to policy language (Social Distance), and an increase in the effectiveness of communicating international policy messages (Communication Effectiveness) in all studied locations (urban, rural and tobacco) and across each of the policy areas (health promotion-general, environment and tobacco control) (1). The greatest impact of the intervention was observed for the general area of 'health promotion' (initially, the most sensitive and unacceptable); and the least effect was noticed for the specific area of 'tobacco control' (initially the most acceptable and the least sensitive subject area).

The impact of the intervention is presented on Figures 2 \& 3 , where the decrease in sensitivity to international policy language (decrease in Social Distance) and the increase in the effectiveness of communicating global policy messages (increase in Communication Effectiveness) achieved as a result of the contextualization, can be seen across all subject area groups (4).
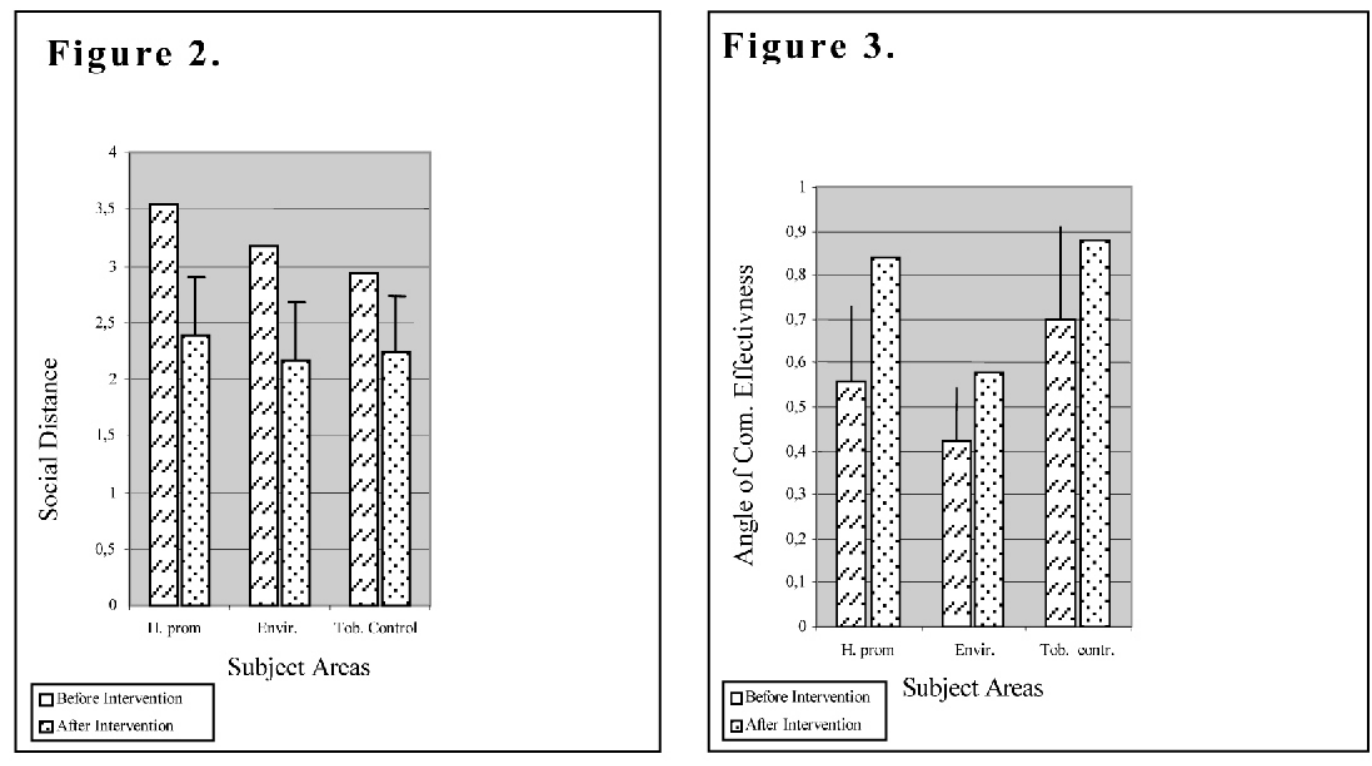

Source: A. Kerekovska (2004)

Figure 2. Social Distance - Pre- and Post-Intervention (by Subject Area)

Figure 3. Communication Effectiveness - Pre-and Post-Intervention (by Subject Area)

councilors from five geographic sites in Bulgaria: Varna (representing the 'urban' type location), Valchi Dol and Bjala (representing the 'rural' type location) and Gotze Delchev and Sandanski (representing the 'tobacco' location. The socio-economic profile, cultural pattern and level of openness to international western influence were the main features upon which the sites selection process was based. The data was collected using a group-interviewing
Such results clearly suggest that by contextually interpreting international health promotion policy and aligning it closely to the cultural patterns of the recipient country, we can increase the acceptability and assure greater effectiveness in communicating global health promotion policy messages at a national level. Moreover, as cultural diversity also embraces all local and sub-state manifestations, the study went further down the vertical route of global policy 
transfer - from the national to the sub-national level. The findings suggested also quite strongly that there is a cultural specificity on a sub-national level that affects the local receptivity to international policy language.

A significant difference was observed between the different locations within Bulgaria, in terms of their level of receptivity (Social Distance) and the effectiveness of communicating (Communication Effectiveness) the existing, as well as the contextualized, health promotion policy messages (1). The lowest sensitivities and the greatest receptivity to the international policy language within all subject areas were found in the urban location (represented by a cosmopolitan city - that is open to international influences and close to the Western ideas); whereas the language for all subject areas proved to be most sensitive and least acceptable in the rather closed rural and tobacco growing areas - politically less internationally influenced and more distant from the western way of thinking. The locations also differed significantly in the impact of the intervention. The smallest increase in the receptivity to global policy messages as a result of the policy language desensitisation was achieved for the urban location, and this occurred across all subject areas. The greatest improvement in the effectiveness of communicating the international health promotion messages was present in those regions most distant from the western culture i.e. rural and tobacco localities. The greatest impact of all came when the translated tobacco control messages were tested in the tobacco growing localities where, with the literal translation and given their economic dependence on tobacco, they were the most sensitive and unreceptive to these policies (4).

These results support the idea that sub-national locations with different socio-economic characteristics and cultural patterns will have varying degrees of tolerance to global health promotion policy messages. Thus, when transferring international policy ideas, apart from the desensitisation and cultural specification of the global policy language to the individual country, a further specification and contextual adaptation might be also required for particular localities in order to improve the communication. This particularly refers to localities that still remain distant even after the policy language has been adapted to the national cultural pattern.

\section{CONCLUSION}

When considering strategies to improve communication of international health promotion policies and ease their implementation into culturally distinctive contexts, it was shown in this study that what is needed is a contextualized interpretation of global policy language to take account of the national cultural specificities. Contextualisation of international policy language increases the possibility of acceptance of ideas locally, potentially enhancing the implementation of health promotion programmes in the Bulgarian context.

As Bulgaria joins the EU in January 2007 this study takes on broader importance. There will be many new policies beyond health to which adjustment is required at a national level; and true citizenship of Europe is dependent upon Bulgarians as a whole being able to identify with the intentions of European policies. Health promotion is only the beginning!

Improving the communication of international policies through cultural specification of policy language might also be important for other countries and localities that are distant from the western values, culture and thinking. Enhancing the transferability of international policy language would create the potential for substantially improved implementation of EU policy into the new members group and the accession states.

\section{REFERENCES}

1. Керековска, А. Оценка на ефективността на пренасяне на международни политически послания за промоция на здраве към конкретен български контекст. Здравна икономика $и$ мениджмънт, 2, 2006, No 20, 22-29.

2. Foot, A. A Model of Communication Effectiveness. Journal of Communication, 1970, No 20, p. 81.

3. Hofstede, G. Cultures and Organisations. Software of the Mind. Intercultural Cooperation and its Importance for Survival. McGraw-Hill Book Company: London, 1996.

4. Kerekovska, A. Strengthening Cross-cultural Policy Transfer: The Case of International Health Promotion and Tobacco Control. PhD Dissertation, University of Glamorgan, Wales, UK, 2004.

5. Sunrcke, M., M. McKee, R. Sauto Arle, S. Tsolova, J. Mortonsen. The Contribution of Health to the Economy of the European Union. Brussels: European Commission, 2005.

6. Warner, M. Lower Socioeconomic Groups and Preventive Public Health Programmes: A Problem of Communication Effectiveness. Canadian Journal of Public Health, 64, 1973, No 6, 562-573.

7. World Health Organisation. Ottawa Charter for Health Promotion. Health Promotion, 1, 1986, No 4, iii-v.

8. World Health Organisation. Adelaide Recommendations on Healthy Public Policy. WHO: Geneva, 1988.

9. World Health Organisation. Jakarta Declaration on Leading Health Promotion into the 21st Century. Health Promotion International, 12, 1997, 261-264. 